\title{
Ultrasound plaque characterisation, genetic markers and risks
}

\author{
Andrew N. Nicolaides ${ }^{1,2}$, Stavros K. Kakkos², Maura Griffin², George Geroulakos², Evy \\ Bashardi ${ }^{1}$ \\ (1) Department of Neurovascular Sciences, The Cyprus Institute of Neurology and Genetics, Nicosia, Cyprus. \\ (2) Department of Vascular Surgery, Faculty of Medicine, Imperial College, London, UK.
}

\section{Key Words}

Ultrasound, atherosclerotic plaques, genetic markers

\begin{abstract}
The arterial wall changes detected by ultrasound are the end result of all risk factors (exogenous, endogenous and genetic) known and unknown and are better predictors of risk than any combination of conventional risk factors. However, ultrasound cannot be used in people younger than 45 because characteristic changes occur after this age. Nevertheless, it can be used in individuals over 45 to identify the genetic risk factors associated with atherosclerosis. The identification of genetic factors will subsequently provide a means of identifying individuals at risk at an early age, even childhood. In addition, knowledge of the genetic abnormalities associated with increased risk in a particular individual will provide a means of targeting prophylactic therapy.
\end{abstract}

Copyright @ 2002 S. Karger AG. Base

\section{Introduction}

Extracranial atherosclerotic disease, known also as atherosclerotic disease of the carotid bifurcation has two main clinical manifestations (a) asymptomatic bruits and (b) cerebrovas- cular syndromes such as amaurosis fugax, transient ischaemic attacks (TIAs) or stroke which are often the result of plaque erosion or rupture with subsequent thrombosis producing occlusion or embolisation [1,2]. Internal carotid artery stenosis, the main consequence of atherosclerotic disease of the carotid bifurcation, remains the single preventable cause of ischaemic stroke.

Recent studies involving angiography, high-resolution ultrasound, thrombolytic therapy, plaque pathology, coagulation studies and more recently molecular biology have implicated atherosclerotic plaque rupture as a key mechanism responsible for the development of cerebrovascular events [35].

Atherosclerotic plaques consist of a lipid-rich core and a fibrous cap, which separates the core from the lumen. The lipid-rich core contains $\mathrm{T}$ cells and lipid-laden macrophages (foam cells), which are derived from blood monocytes [3]. T cells produce interferon- $\gamma$, which suppresses the production of collagen by the smooth muscle cells [4] and stimulates the macrophages to produce metalloproteinases (stromelysins, gelatinases, collagenases), which digest existing collagen and other extracellular matrix components. In addition foam cells produce tissue factor, which stimulates thrombus formation when in contact with blood after plaque rupture.

Rupture prone plaques tend to have a large lipid core, a thin fibrous cap, few smooth muscle cells and an abundance of macrophages [5]. Ruptured plaques heal or enlarge by incorporating the thrombus formed on their surface. Some thrombi grow and occlude the lumen or produce emboli. Whether a

\section{KARGER \\ Fax +4161306 1234 \\ E-Mail karger@karger.ch}

www.karger.com (c) 2002 S. Karger AG. Basel

$1424-8832 / 02 / 0326-0371 \$ 18.50 / 0$

Accessibile online at: www.karger.com/journals/pht
Professor A N Nicolaides

The Cyprus Institute of Neurology and Genetics

PO Box 23462

1683 Nicosia - Cyprus

Tel: +357-22-392600 - Fax: +357-22-358237

Email: anicolai@cing.ac.cy 
thrombus will occur or enlarge depends on the local blood flow and the hypercoagulable state.

Conventional arteriography has been used for several decades to investigate the presence and severity of internal carotid artery stenosis. Because it is invasive it cannot be repeated frequently and carries a risk of stroke of $1.2 \%$; in addition angiography provides little information on plaque structure. The development and continuing technical improvement of non-invasive high-resolution vascular ultrasound has enabled us to study the presence, rate of progression or regression of plaques and most importantly their consistency. The ultrasonic characteristics of unstable (vulnerable) plaques have been determined [6,7] and populations or individuals at increased risk for cardiovascular events can now be identified [8]. In addition, high-resolution ultrasound has enabled us to identify the different ultrasonic characteristics of unstable carotid plaques associated with amaurosis fugax, TIAs, stroke and different patterns of CT-brain infarction [6,7]. This information has provided new insight into the pathophysiology of the different clinical manifestations of extracranial atherosclerotic cerebrovascular disease using noninvasive methods.

It has now been realised that there are many unknown risk factors for cardiovascular disease. The last few years have seen the emergence of some new ones such as oestrogen deficiency, hyper-homocysteinaemia, abnormal levels and function of fibrinogen, TPA, PAI-1, factor V, XII, VII and XIII, Lipoprotein(a) and C-reactive protein. In addition a number of cardiovascular genes have been identified with protective or harmful polymorphisms.

Some of the cardiovascular genes may influence lipid metabolism and the early formation of atherosclerotic plaques. Others may influence the rate of progression of plaques (rapid or slow) and yet others may determine whether plaques will be stable containing an abundance of collagen or unstable having a large lipid pool and a thin fibrous cap. Unstable plaques tend to ulcerate. Whether healing or thrombosis will subsequently occur is also influenced by the hypercoagulable state and thrombophilic genotypes.

The aim of this presentation is to highlight the advances of high-resolution ultrasound on carotid plaque characterisation, its clinical importance and the contribution of genetic epidemiology in understanding the basic pathophysiology involved.

\section{The need for $\beta$-mode image normalisation - description of the method}

High-resolution ultrasound provides information not only on the degree of carotid artery stenosis but also on the characteristics of the arterial wall including the size and consistency of the atherosclerotic plaque. Different classifications have been proposed in the literature, according to plaque consisten- cy, resulting in considerable confusion. For example, plaques containing medium or high-level uniform echoes were classified as homogenous by Reilly [9] and correspond closely to Johnson's dense and calcified plaques, [10] to Gray-Weale's type 3 and 4 [11] and to Widder's type I and II plaques [12] (i.e. echogenic or hyperechoic). A recent consensus on carotid plaque characterisation has suggested that echodensity should reflect the overall brightness of the plaque with the term hyperechoic referring to echogenic and the term hypoechoic referring to echolucent plaques [13]. The reference structure, to which plaque echodensity should be compared with, is for hypoechoic plaques, blood; for the isoechoic, the sternomastoid muscle; and for the hyperechoic ones, the bone of the adjacent cervical vertebrae.

Measurements of texture should not be confused with measurements of echodensity. The term homogenous should refer to plaques of uniform consistency irrespective of whether they are predominantly hypoechoic or hyperechoic. The term heterogenous should be used for plaques of non-uniform consistency, i.e. having both hypoechoic and hyperechoic areas. Although this may appear to be a relatively simplistic point of view, it is the basis for a standard classification of carotid plaques based on high-resolution ultrasound.

There is enough evidence published to support the clinical usefulness of ultrasonic plaque characterisation, patients with hypoechoic carotid plaques being at increased risk of stroke. Polak has recently investigated the association between stroke and internal carotid artery plaque echodensity [14]. In this study plaques were subjectively characterised as hypoechoic, isoechoic or hyperechoic in relation to the surrounding soft tissues. The stroke rate for hypoechoic plaques was 2.78 times higher than for isoechoic and hyperechoic plaques. The authors suggested that quantitative methods of grading carotid plaque echomorphology such as computer-assisted plaque characterisation, developed by our group, might be more precise in determining the association between hypoechoic (echolucent) plaques and the incidence of stroke.

Computer assisted plaque characterisation involves processing of digitised B-mode images of plaques taken from a duplex scanner with fixed instrument settings including gain and time control. The median of the frequency distribution of grey values of the pixels within the plaque (grey scale medianGSM, scale $0-255,0=$ black, $255=$ white) is used as the measurement of echodensity. Early work has demonstrated that plaques with a GSM of less than 32, i.e. echolucent plaques have been found to have a five-fold increase in the prevalence of silent brain infarcts on CT-brain scans [15]. Soon it became apparent that ultrasonic image normalisation was necessary, so that images captured under different instrument settings, from different scanners, by different operators and through different peripherals such as video or magneto-optical disk could be comparable.

As a result a method has been developed to normalise 
images by means of digital image processing using blood and adventitia as the two reference points.[16] With the use of commercially available software (Adobe Photoshop version 3.0 or later, Adobe Systems Inc.) and the "histogram" facility, the gray scale median (GSM) of the 2 reference points (blood and adventitia) in the original B-mode image is determined. Algebraic (linear) scaling of the image is performed with the "curves" option of the software so that in the resultant image the GSM of blood equals 0 to 5 and that of the adventitia equals 185 to 195 . Thus brightness of all pixels in the image including those of the plaque becomes adjusted according to the two reference points. This results in a significant improvement in the comparability of the ultrasonic tissue characteristics [16-18].

Accurate selection of the appropriate areas of blood and adventitia for image normalisation and the avoidance of areas of acoustic shadow in the selection of the plaque area are imperative. The duplex settings recommended are as follows: Maximum dynamic range, low persistence and high frame rate. A high frequency linear array transducer ideally 7-10 $\mathrm{MHz}$ should be used. A high dynamic range ensures a greater range of grey scale values. High frame rate ensures good temporal resolution. In addition to these pre-sets the time gain compensation curve should be positioned vertically through the lumen of the vessel, as there is little attenuation of the beam at this point. The overall gain should be adjusted to give optimum image quality (bright echoes with minimum noise in the blood). A linear post-processing curve should also be used and finally where possible the ultrasound beam should be at 90 degrees to the arterial wall.

The previously discussed guidelines should result in the following:

- An area of noiseless blood

- An echodense piece of adventitia in the vicinity of the plaque and

- Visualisation of the extent and borders of the plaque. It is here that colour images can provide further information about plaque outline.

Three major reproducibility studies have been performed in order to establish the validity of the method of image normalisation and the value of GSM measurements [16-18]. These three studies have demonstrated that GSM after image normalisation is a highly reproducible measurement that could be used in natural history studies of asymptomatic carotid atherosclerotic disease, aiming to identify patients at higher risk of stroke.

It should be pointed out that training is essential if the level of reproducibility reported above is to be achieved. Training is necessary not only in the use of the software but also in the appropriate scanning technique. For an experienced ultrasonographer training requires two days.

\section{Clinical significance of carotid plaque echodensity and structure}

The clinical importance of ultrasonic plaque characterisation could be focused on three main areas. First, cross-sectional studies aiming at better understanding of the pathophysiology of carotid disease. Second, natural history studies seeking to identify high and low risk groups for stroke; these studies should refine the indications on selection of symptomatic or asymptomatic patients not only for carotid endarterectomy but also for stenting. Third, ultrasonic carotid plaque characterisation can provide useful information for the endovascular specialist who is likely to use it to select patients for carotid angioplasty and stenting (CAS) [19].

\section{Cross-sectional studies}

The use of the above method of image normalisation and analysis has resulted in the identification of differences in carotid plaque structure - in terms of echodensity and degree of stenosis - not only between symptomatic and asymptomatic plaques but also between plaques associated with retinal and hemispheric symptoms [7]. In asymptomatic and symptomatic patients having amaurosis fugax, TIAs and stroke with good recovery having 50-99\% stenosis on carotid duplex scan, plaques associated with symptoms were significantly more hypoechoic, with higher degrees of stenosis than those not associated with symptoms (mean GSM $=13.3$ versus 30.5 and mean degree of stenosis $=80.5 \%$ versus $72.2 \%$ ). Furthermore, plaques associated with amaurosis fugax were hypoechoic (mean GSM = 7.4) and severely stenotic (mean stenosis $85.6 \%$ ). Plaques associated with TIAs and stroke had a similar echodensity and a similar degree of stenosis (mean GSM = 14.9 versus 15.8 and degree of stenosis $=79.3 \%$ versus $78.1 \%$ ). These findings confirm previous reports, which have shown that hypoechoic plaques are more likely to be associated with symptoms $[15,16,20]$. In addition they support the hypothesis that amaurosis fugax has a different pathophysiological mechanism to that of TIAs and stroke.

Our group has found that GSM separates echomorphologically the carotid plaques associated with silent nonlacunar CTdemonstrated brain infarcts from plaques that are not so associated. The median GSM of plaques associated with ipsilateral nonlacunar silent CT-demonstrated brain infarcts was 14 , and that of plaques that were not so associated was 30 ( $p=0.003$ ) [18]. Additionally, emboli counted on transcranial Doppler (TCD) in the ipsilateral middle cerebral artery were more frequent in the presence of low-plaque echodensity (low GSM), but not in the presence of a high degree of stenosis. These data support the embolic nature of cerebrovascular symptomatology [21].

The role of biomechanical forces in the induction of plaque 
fatigue and rupture has been emphasised [22-24]. In our group of patients, carotid plaques associated with amaurosis fugax were hypoechoic and were associated with very high-grade stenoses. It may well be that the plaques that are hypoechoic and homogenous, undergo low internal stresses and therefore do not rupture but progress to tighter stenosis with post-stenotic dilatation, turbulance, platelet adhesion in the post-stenotic area resulting in the eventual production of showers of small platelet emboli. Such small platelet emboli may be too small to produce hemispheric symptoms but are detected by the retina. In contrast plaques associated with TIAs and stroke were less hypoechoic and less stenotic than those associated with amaurosis fugax. These plaques are hypoechoic but more heterogenous undergoing stronger internal stresses. Therefore, they may tend to rupture at an earlier stage (lower degrees of stenosis), producing larger particle debris (plaque constituents or thrombi) that deprives large areas of the brain of adequate perfusion.

\section{Natural history studies}

The first study, which has shown the value of ultrasonic characterisation of carotid bifurcation plaques in asymptomatic patients, was done by Johnson in the early 80's.[10] In that study, hypoechoic carotid plaques in comparison with the hyperechoic or calcified ones increased the risk of stroke during a follow-up period of 3 years; this effect was prominent in patients with carotid stenosis more than $75 \%$ (as estimated by cross-sectional area calculations and spectral analysis), as stroke occurred in $19 \%$ of them. None of the patients with calcified plaques developed a stroke.

A second study performed in the 80's by Sterpetti [25], has shown that the severity of stenosis (lumen diameter reduction greater than $50 \%$, haemodynamic stenosis) and the presence of a heterogenous plaque were both independent risk factors for the development of new neurological deficits (TIA and stroke). Twenty seven percent of the patients with heterogenous plaques and haemodynamically significant stenosis developed new symptoms. Unfortunately, their study had mixed cases as $37 \%$ of the patients had a history of previous neurologic symptoms, mainly hemispheric ones. History of these neurologic symptoms was a risk factor for the development of new neurological symptoms during the follow-up period, although this was found only in the univariate analysis. Because no subgroup analysis was performed, no conclusion can be drawn regarding asymptomatic or symptomatic patients.

The last study published in the 80's by Langsfeld [26] confirmed that patients with hypoechoic plaques (type 1, predominantly echolucent raised lesion, with thin "egg shell" cap of echogenicity and type 2, echogenic lesions with substantial areas of echolucency) had a twofold risk of stroke of $15 \%$ in comparison with $7 \%$ of those having hyperechoic plaques (type 3, predominately echogenic with small area(s) of echolucency deeply localised and occupying less than a quarter of the plaque and type 4, uniformly dense echogenic lesions). Patients also with $>75 \%$ stenosis, were at increased risk. However, the overall incidence of new symptoms was low, in contrast with the previous studies, perhaps because only asymptomatic patients were included in that study. Based on their results, the authors proposed an aggressive approach in those patients with $>75 \%$ stenosis and heterogenous plaques. There is some confusion regarding the interchangeable use of the terms heterogenous and hypoechoic in that article. Additionally the authors raised the point that it is important for each laboratory to verify its ability to classify plaque types. The same group in another study published four years later reported a 5.7\% annual vessel event rate (TIA and stroke) for echolucent carotid plaques versus $2.4 \%$ for the echogenic ones $(\mathrm{p}=0.03)[27]$.

Given the fair interobserver reproducibility for type 1 plaques, the use of reference points was proposed: anechogenicity to be standardized against circulating blood, isoechogenicity against sternomastoid muscle and hyperechogenicity against bone (cervical vertebrae). A similar method has been used in the late 1990's by Polak [14], who investigated the association between stroke and internal carotid artery plaque echodensity in 4886 asymptomatic individuals aged 65 years or older, who were followed up prospectively for 48 months. Some $68 \%$ of those had carotid artery stenosis, which exceeded $50 \%$ in 270 patients. In this study plaques were subjectively characterised as hypoechoic, isoechoic or hyperechoic in relation to the surrounding soft tissues. Hypoechoic plaques causing stenoses $50-100 \%$ were associated with a significantly higher incidence of ipsilateral, non-fatal stroke than iso- or hyperechoic plaques of the same degree of stenosis (relative risk 2.78 and 3.08 respectively). The authors of this study suggested that quantitative methods of grading carotid plaque echomorphology such as computer-assisted plaque characterisation might be more precise in determining the association between hypoechoic (echolucent) plaques and the incidence of stroke.

The Troms $\emptyset$ study conducted in Norway in 223 subjects with carotid stenosis $>35 \%$ has also found that subjects with echolucent atherosclerotic plaques have increased risk of ischaemic cerebrovascular events independent of degree of stenosis.[28] The authors give no details on patient's neurological history. The adjusted relative risk for all cerebrovascular events in subjects with echolucent plaques was $4.6(95 \%$ c.i. 1.1 to 18.9 ), and there was a significant linear trend $(\mathrm{p}=0.015)$ for higher risk with increasing plaque echolucency. Ipsilateral neurological events were also more frequent in patients with echolucent or predominantly echolucent plaques (17.4\% and $14.7 \%$, respectively). The authors concluded that evaluation of plaque morphology in addition to the grade of stenosis might improve clinical decision-making and differen- 
tiate treatment for individual patients and that computer-quantified plaque morphology assessment, being a more objective method of ultrasonic plaque characterisation, may further improve this.

This method has been recently used by Grønholdt,[29] who found that echolucent plaques causing $>50 \%$ diameter stenosis were associated with risk of future stroke in symptomatic $(\mathrm{n}=135)$ but not asymptomatic $(\mathrm{n}=111)$ individuals. Echogenicity of carotid plaques was evaluated with high-resolution B-mode ultrasound and computer-assisted image processing. The mean of the standardised median gray-scale values of the plaque was used to divide plaques into echolucent and echorich. Relative to symptomatic patients with echorich $50 \%$ to $79 \%$ stenotic plaques, those with echorich $80 \%$ to $99 \%$ stenotic plaques, echolucent $50 \%$ to $79 \%$ stenotic plaques, and echolucent $80 \%$ to $99 \%$ stenotic plaques had relative risks of ipsilateral ischaemic stroke of 3.1 (95\% c.i., 0.7 to 14), 4.2 (95\% c.i., 1.2 to 15 ), and 7.9 (95\% c.i., 2.1 to 30 ), equivalent to absolute risk increase of $11 \%, 18 \%$, and $28 \%$, respectively. The authors suggested that measurement of echolucency, together with degree of stenosis, might improve selection of patients for carotid endarterectomy. The relatively small number of asymptomatic individuals was probably the reason explaining why plaque characterisation was not helpful in predicting risk in the asymptomatic group.

\section{Genetic Epidemiology of Vascular Disease}

One of the earliest identified and studied genes is the apolipoprotein E. ApoE which plays a central role in the regulation of lipid metabolism by mediating the binding of lipoprotein particles to the LDL receptor and ApoE(remnant) receptor. The ApoE gene has three alleles; E2, E3 and E4. These alleles interact differently with lipoprotein receptors and alter circulating lipid levels. Although $60 \%$ of variation in plasma cholesterol is genetically determined, ApoE polymorphism accounts for only $14 \%$ of this. This illustrates the important effect of other genes in lipid metabolism.

The frequency of the ApoE alleles in the European populations is as follows: E3 80\%, E2 8\% and E4 12\%. The E2 allele is associated with low LDL, high triglycerides and low HDL. The E4 allele is associated with high LDL, high triglycerides and low HDL. Kinetic studies have shown that E4 allows the accumulation of LDL and E2 the accumulation of triglycerides. The presence of an E4 allele increases the risk of coronary heart disease and the genotype E3/E4 is associated with myocardial infarction at an early age [30]. In young men, under 40 years, undergoing coronary angioplasty the E4/E4 genotype was 16 times more common than in the general population [31]. The frequency of the E4 allele is increased in patients with ischaemic cerebrovascular disease [32] and in a recent case control study the E2 allele was associated with pro- tection from stroke at age 70-79 but not in those over 80 years. Individuals not carrying an E2 allele had a relative risk of 2.6 for stroke [33].

Another recent case control study has investigated the frequency of the ApoE and beta-fibrinogen G/A-455 polymorphisms in ischaemic stroke [34]. After excluding intracranial haemorrhage, 277 patients with focal cerebral ischaemia were compared with 277 normal controls. There was no difference in the allele frequency between the groups but when stroke was classified according to type, the E4 allele and the A allele of the beta-fibrinogen genes were more frequent in those with stroke $(n=70)$ due to large vessel disease. The key message is that clinical end-points can be the result of different pathologies and future studies should use end-points that are appropriate for the genes studied.

The realisation that clinical end-points can be the result of different pathologies has stimulated many research teams to use ultrasound in an attempt to produce better defined phenotypes. It has been demonstrated that carriers of E4 allele have carotid wall thickness greater than subjects with the E3 allele.[35] The Q451 allele of the CETP gene is associated with increased plasma CETP and lower carotid wall thickness.[36] This is an example of a protective gene polymorphism. The LL genotype of the PON1 gene is associated with presence and severity of carotid atherosclerosis on duplex (independent risk factor)[37] and so does the Hind III genotype of the lipoprotein lipase gene [38]. Individuals with carotid stenosis greater than $75 \%$ have a higher frequency $(47 \%)$ of the $\mathrm{V}$ allele (A/V polymorphism) of the MTHFR gene than controls $(27 \%)$ with carotid stenosis less than $25 \%$ [39]. The TT genotype of the p-fibronogen gene (C148T of polymorphism) is associated with the highest grades of carotid stenosis on duplex [40]. The D allele of the ACE gene (I/D polymorphism) and the $\mathrm{D} / \mathrm{D}$ genotype are more frequent in patients with carotid stenosis or ischaemic cerebrovascular disease (independent risk factor) [41, 42].

Case control studies such as the above provide information on associations and do not allow inferences on causality. Only hypotheses can be made. What is needed now is prospective studies in which both clinical risk factors, ultrasound and genetic markers are included. With adequate follow-up of patients the causative link between molecular mechanisms and the effect of established risk factors on the formation, progression, type of and rupture of plaques and to clinical events can be obtained. This is our new challenge. 


\section{References}

1. Gutstein DE, Fuster V. Pathophysiology and clinical significance of atherosclerotic plaque rupture. Cardiovasc Res 1999;41:323-333.

2. Zukowski AJ, Nicolaides AN, Lewis RT, Mansfield AO, Williams MA, Helmis E, Malouf GM, Thomas D, Al-Kutoubi A, Kyprianou P, et al. The correlation between carotid plaque ulceration and cerebral infarction seen on CT scan. J Vasc Surg 1984;1:782786.

3. Libby P. Molecular basis of acute coronary syndromes. Circulation 1995;91:2844-2850.

4. Clinton S, Underwood R, Hayes L, Sherman ML, Kufe DW, Libby P. Macrophage-colony stimulating factor gene expression in vascular cells and human atherosclerosis. Am J Pathol 1992; 140:301-316.

5. Davies MJ, Richardson PD, Woolf N, Katz DR, Mahn J. Risk of thrombosis in human atherosclerotic plaques: role of extracellular lipid, macrophage and smooth muscle cell content. Br Heart J 1993;69:377-381.

6. Sabetai MM, Tegos TJ, Nicolaides AN, ElAtrozy TS, Dhanjil S, Griffin M, Belcaro G, Geroulakos G. Hemispheric symptoms and carotid plaque echomorphology. J Vasc Surg 2000;31:39-49.

7. Tegos TJ, Sabetai MM, Nicolaides AN, ElAtrozy TS, Dhanjil S, Stevens JM. Patterns of brain computed tomography infarction and carotid plaque echogenicity. J Vasc Surg 2001;33:334-339.

8. Belcaro G, Nicolaides AN, Laurora G, Cesarone MR, De Sanctis M, Incandela L, Barsotti A . Ultrasound morphology classification of the arterial wall and cardiovascular events in a 6-year follow-up study. Arterioscler Thromb Vasc Biol 1996;16:851-856.

9. Reilly LM, Lusby RJ, Hughes L, Ferrell LD, Stoney RJ, Ehrenfeld WK. Carotid plaque histology using real-time ultrasonography: clinical and therapeutic implications. Am J Surg 1983;146:188-193.

10. Johnson JM, Kennelly MM, Decesare D, Morgan S, Sparrow A. Natural history of asymptomatic carotid plaque. Arch Surg 1985;120:1010-1012.

11. Gray-Weale AC, Graham JC, Burnett JR, Burne K, Lusby RJ. Carotid artery atheroma: comparison of preoperative B-mode ultrasound appearance with carotid endarterectomy specimen pathology. J Cardiovasc Surg 1988;29:676-681.

12. Widder B, Paulat K, Hachspacher J, Hamann $\mathrm{H}$, Hutschenreiter S, Kreutzer C, Ott F, Vollmar J. Morphological characterization of carotid artery stenoses by ultrasound duplex scanning. Ultrasound Med Biol 1990;16:349354.

13. deBray JM, Baud JM, Dauzat M for the Consensus Conference: Concensus on the morphology of carotid plaques. Cerebrovasc Dis 1997;7:289-296.

14. Polak JF, Shemanski L, O'Leary DH, Lefkowitz D, Price TR, Savage PJ, Brant WE, Reid C. for the Cardiovascular Health Study. Hypoechoic plaque at US of the carotid artery: An independent risk factor for incident stroke in adults aged 65 years or older. Radiology 1998;208:649-654

15. El-Barghouty NM, Nicolaides A, Bahal V, Geroulakos G, Androulakis A. The identification of high risk carotid plaque. Eur J Vasc Surg 1996;11:470-478.

16. El-Atrozy T, Nicolaides A, Tegos T, Zarka AZ, Griffin M, Sabetai M. The effect of B-mode image standardisation on the echodensity of symptomatic and asymptomatic carotid bifurcation plaques. Int Angiol 1998;17:179-186.

17. Tegos TJ, Sabetai MM, Nicolaides AN, Pare G, Elatrozy TS, Dhanjil S, Griffin M Comparability of the ultrasonic tissue characteristics of carotid plaques. J Ultrasound Med 2000; 19:399-407.

18. Sabetai MM, Tegos TJ, Nicolaides AN, Dhanjil S, Pare GJ, Stevens JM. Reproducibility of computer-quantified carotid plaque echogenicity. Stroke 2000;31:2189-2196.

19. Reid DB. Carotid plaque characterization: Helpful to endarterectomy and endovascular surgeons. J Endovasc Surg 1998;5:247-250.

20. Geroulakos G, Ramaswami G, Nicolaides A James K, Labropoulos N, Belcaro G, Holloway M. Characterisation of symptomatic and asymptomatic carotid plaques using high resolution real-time ultrasonography. $\mathrm{Br} \mathrm{J}$ Surg 1993;80:1274-1277.

21. Tegos TJ, Sabetai MM, Nicolaides AN, Robless P, Kalodiki E, Elatrozy TS, Ramaswami G, Dhanjil S. Correlates of embolic events detected by means of transcranial Doppler in patients with carotid atheroma. J Vasc Surg 2001;33:131-138

22. Richardson PD, Davies MJ, Born GVR. Influence of plaque configuration and stress distribution and fissuring of coronary atherosclerotic plaque. Lancet 1989;ii:941-944.

23. Ku DN, McCord BN. Cyclic stress causes rupture of the atherosclerotic plaque cap. Circulation 1993;88(Suppl 1):1362 (abst).

24. Glagov S, Bassiouny HS, Sakaguchi Y, Goudet CA, Vito RP. Mechanical determinants of plaque modeling, remodeling and disruption. Atherosclerosis 1997;131(Suppl):S13-S14.

25. Sterpetti AV, Schultz RD, Feldhaus RJ, Davenport KL, Richardson M, Farina C, Hunter WJ . Ultrasonographic features of carotid plaque and the risk of subsequent neurologic deficits. Surgery 1988;104:652-660.

26. Langsfeld M, Gray-Weale AC, Lusby RJ. The role of plaque morphology and diameter reduction in the development of new symptoms in asymptomatic carotid arteries. J Vasc Surg 1989;9:548-557.

27. Bock RW, Gray-Weale AC, Mock PA, Robinson DA, Irwig L, Lusby RJ. The natural history of asymptomatic carotid artery disease. J Vasc Surg 1993;17:160-171.

28. Mathiesen EB, Bønaa KH, Joakimsen O. Echolucent Plaques Are Associated With High Risk of Ischemic Cerebrovascular Events in Carotid Stenosis. The Troms $\emptyset$ Study. Circulation. 2001;103:2171-2175.

29. Grønholdt M-LM, Nordestgaard BG Schroeder TV, Vorstrup S, Sillesen H. Ultrasonic echolucent carotid plaques predict future strokes. Circulation 2001;104:68-73.

30. Vauhkonen I, Niskanen L, Ryynanen M, Voutilainen R, Partanen J, Toyry J, Mercuri M, Rauramaa R, Uusitupa M. Divergent association of apolipoprotein E polymorphism with vascular disease in patients with NIDDM and control studies. Diabet Med 1997;14:748-756.

31. van Bockxmeer FM, Mamotte CD. Apolipoprotein epsilon 4 homozygocity in young men with coronary heart disease. Lancet 1992;340:879-880.

32. Pedro-Botet S, Senti M, Nogues X, RubiesPrat J, Roquer J, D’Olhaberriague L, Olive J. Lipoprotein and apolipoprotein profile in men with ischemic stroke: role of lipoprotein (a), triglyceride-rich lipoproteins and apolipoprotein E polymorphism. Stroke 1992;23:15561562.

33. Ferrucci L, Guralnik JM, Pahor M, Harris T, Corti MC, Hyman BT, Wallace RB, Havlik RJ. Apolipoprotein E, E2 allele and risk of stroke in the older population. Stroke 1997;28:24102416.

34. Kessler C, Spitzer C, Stauske D, Mende S, Stadlmuller J, Walther R, Rettig R. The apolipoprotein $\mathrm{E}$ and beta-fibrinogen G/A-455 gene polymorphisms are associated with ischaemic stroke involving large vessel disease. Arterioscler Thromb Vasc Biol 1997; 17:2880-2884

35. Cattin L, Fisicaro M, Tonizzo M, Valenti M, Danek GM, Fonda M, D Col PG, Casagrande S, Pincetri E, Bovenzi M, Baralle F. Polymorphism of the apolipoprotein $\mathrm{E}$ gene and early carotid atherosclerosis defined by ultrasonography in asymptomatic adults. Arterioscl Thromb Vasc Biol 1997;17:91-4.

36. Kakko S, Tamminen M, Paivansalo M, Kauma H, Rantala AO, Lilja M, Reunanen A, Kesaniemi YA, Savolainen MJ. Cholesteryl ester transfer protein gene polymorphisms are associated with carotid atherosclerosis in men. Eur J Clin Invest 2000;30:18-25.

37. Schmidt H, Schmidt R, Niederkom K, Gradert A, Schumacher M, Watzingen N, Hartung HP, Kostner GM. Paraoxonase PON1 polymorphism leu-Met54 is associated with carotid atherosclerosis: results of the Austrian Stroke Prevention Study. Stroke 1998;29:2043-8.

38. Chen L, Patsch W, Boerwinkle E. HindIII DNA polymorphism in the lipoprotein lipase gene and plasma lipid phenotypes and carotid artery atherosclerosis. Hum Genet 1996; 98:551-6.

39. Bova I, Chapman J, Sylantiev C, Korczyn AD, Bornstein NM. The A677V MTHFR gene polymorphism and carotid atherosclerosis. Stroke 1999;30:2180-2

40. Schmidt H, Schmidt R, Niederkom K, Horner S, Becsagh P, Reinhart B, Schumacher M, Weinrauch V, Kostner GM. Beta-fibrinogen gene polymorphism (C148T) is associated with carotid atherosclerosis: results of the Austrian Stroke Prevention Study. Arterioscler Thromb Vasc Biol 1998; 18:487-92.

41. Pfohl M, Fetler M, Koch M, Barth CM, Rudiger W, Haring HU. Association between angiotensin I-converting enzyme deletion alle- 
les were not associated with increased wall thickness of carotid and femoral arteries in healthy subjects from the Stanislas cohort. Atherosclerosis 1998;140:89-95.
42. Kostulas K, Huang WX, Crisby M, Jin YP, He B, Lannfelt L, Eggertsen G, Kostulas V, Hillert J. An angiotensin-converting enzyme gene polymorphism suggests a genetic distinction between ischaemic stroke and carotid stenosis. Eur J Clin Invest 1999;29:478-83. 